\title{
From quantum transitions to electronic motions
}

\author{
Ferenc Krausz ${ }^{1,2}$ (1)
}

Received: 9 September 2016 / Accepted: 4 November 2016 / Published online: 15 December 2016

(C) The Author(s) 2016. This article is published with open access at Springerlink.com

\begin{abstract}
Laser spectroscopy and chromoscopy permit precision measurement of quantum transitions and captures atomic-scale dynamics, respectively. Frequency- and time-domain metrology ranks among the supreme laser disciplines in fundamental science. For decades, these fields evolved independently, without interaction and synergy between them. This has changed profoundly with controlling the position of the equidistant frequency spikes of a mode-locked laser oscillator. By the self-referencing technique invented by Theodor Hänsch, the comb can be coherently linked to microwaves and used for precision measurements of energy differences between quantum states. The resultant optical frequency synthesis has revolutionized precision spectroscopy. Locking the comb lines to the resonator round-trip frequency by the same approach has given rise to laser pulses with controlled field oscillations. This article reviews, from a personal perspective, how the bridge between frequency- and time-resolved metrology emerged on the turn of the millennium and how synthesized severalcycle laser fields have been instrumental in establishing the basic tools and techniques for attosecond science.
\end{abstract}

Dedicated to the 75th birthday of Theodor W. Hänsch.

This article is part of the topical collection "Enlightening the World with the Laser" - Honoring T. W. Hänsch guest edited by Tilman Esslinger, Nathalie Picqué, and Thomas Udem.

Ferenc Krausz

Ferenc.Krausz@mpq.mpg.de

1 Max-Planck-Institut für Quantenoptik, Hans-Kopfermann-Strasse 1, 85748 Garching, Germany

2 Fakultät für Physik, Ludwig-Maximilians-Universität, Am Coulombwall 1, 85748 Garching, Germany
The frequency of microwave to optical radiation absorbed or emitted upon electronic transitions between quantum states of atoms has been the most accurately measured physical quantity to date, allowing to put the laws of quantum physics to a test, determine fundamental constants (and their possible drifts), and define the standards for time and length [1]. Precision spectroscopy underlying these applications relies on excited quantum states the energy of which (with respect to the ground state) is precisely defined. Heisenberg's uncertainty principle dictates that such states decay on very long timescales (by atomic standards). Accurate measurement of this energy requires highly stationary sinusoidal waves consisting of trillions to quadrillions of identical cycles.

By sharp contrast, wavepackets formed by the superposition of stationary quantum states evolve on ultrafast timescales. Nuclear wavepacket motions, in terms of which quantum mechanics describes molecular dynamics of any kind, occur on a timescale of several to several hundred femtoseconds ( $1 \mathrm{fs}=10^{-15} \mathrm{~s}$ ), see Ref. [2], whereas electronic wavepacket dynamics underlying any dynamic change of electronic structure unfolds over tens to thousands of attoseconds ( 1 as $=10^{-18}$ s), see Ref. [3]. Observing and controlling dynamic changes of molecular and electronic structure in real time therefore call for femtosecond- and attosecond-duration pulses and femtosecond- and attosecond-varying forces, respectively.

From these considerations, it appears plausible that measuring quantum transitions and capturing dynamics require very different forms of radiation, with seemingly little room for any synergies. Hence, not very surprisingly, the fields of precision laser spectroscopy and ultrafast laser science evolved independently over decades with little interaction or even communication between the respective scientific communities. This state of affairs changed 
radically on the turn of millennium, thanks to gaining full control over the series of equidistant eigenfrequencies (henceforth, frequency comb) of a mode-locked laser by Theodor Hänsch and his coworkers, Ronald Holzwarth and Thomas Udem. In doing so, they have created a clockwork able to count optical field oscillations of more than $10^{15}$ cycles per second. Their method dramatically simplified and improved the instrumentation for high-precision optical spectroscopy and opened the door for the development of next-generation clocks based on optical quantum transitions [4-15].

The resultant optical frequency synthesis also led to the first fully controlled femtosecond pulses from a modelocked laser, controlled not only in terms of their pulse envelope, but also in terms of their field oscillations. These advances have been instrumental in creating the ability to observe and control atomic-scale electronic motions in real time [3]. Here, I pay tribute to these advances from a personal perspective, without attempting a comprehensive review and by referring only to a few selected references thought to be helpful to readers seeking deeper insight. For a more complete survey of relevant literature, the reader is kindly referred to Ref. [3].

\section{Time-resolved metrology meets frequency-domain metrology}

I felt greatly honored when I was invited to give a colloquium at the Max-Planck-Institute of Quantum Optics as a young postdoc in 1995. Our group in Vienna just finished a study of the non-stationary nature of the pulses emerging from locking the longitudinal modes of a laser (in our case: Ti:sapphire laser) over a bandwidth of nearly $100 \mathrm{THz}$, implying a pulse duration of less than 10 femtosecond $(<4$ wave cycles). We found that the phase $\varphi$ of the carrier wave with respect to the pulse envelope of the electric field,

$\mathbf{E}_{L}(t)=(1 / 2) \mathbf{E}_{\text {peak }} a_{L}(t) e^{-i\left(\omega_{L} t+\varphi\right)}+c . c$.

where $\mathbf{E}_{\text {peak }}, a_{L}(t)$, and $\omega_{L}$ stand for the electric field vector at the pulse peak, the normalized complex amplitude, and the (angular) carrier frequency of the laser field $\mathbf{E}_{L}(t)$, respectively, was slipping all the time from one pulse to the next in the output pulse train even in an apparently perfectly stationary mode of operation.

We devised a scheme that allowed us to measure this phase shift $\Delta \varphi$ occurring upon each round trip in the cavity and its dependence on the linear and nonlinear properties of the laser resonator. I concluded the presentation of these results 16 with arguing that gaining control over this phase slippage would be of tremendous value for the study of strong-field-induced electron processes, enabling their control within the optical field cycle, i.e., on an attosecond timescale, which might open the door for the extension of time-resolved science to real-time observation and control of the electrons' atomic-scale motion.

Subsequent illuminating discussions with Theodor Hänsch, Thomas Udem, and coworkers ignited a collaboration between our groups which continues to this day and culminated - on the turn of the new millennium-in the generation of strong waveform-controlled light and its use for controlling attosecond electron processes $[17,18]$.

\section{Laser mode locking: in the time and frequency domain}

Our study in 1996 revealed that a continuous-wave modelocked laser does not deliver identical pulses, no matter how perfectly its longitudinal modes are phase locked to each other and how perfectly they are equidistantly spaced in frequency. This can be intuitively understood by considering that a laser resonator always contains some dispersive components, in which the group and phase velocities, determining the propagation speed of the pulse envelope and the carrier wave, respectively, are (slightly) different. This gives rise to a carrier wave shifting with respect to the pulse envelope by

$\Delta \varphi=\phi_{R}-\omega_{L} T_{R}$

upon each round trip in the laser resonator, where $\phi_{R}$ is the round-trip phase delay (determined by the phase velocity) and $T_{R}$ is the round-trip time of the laser pulse (determined by the group velocity), see Fig. 1a. A hypothetical sensor measuring the laser field at any (arbitrarily chosen) position

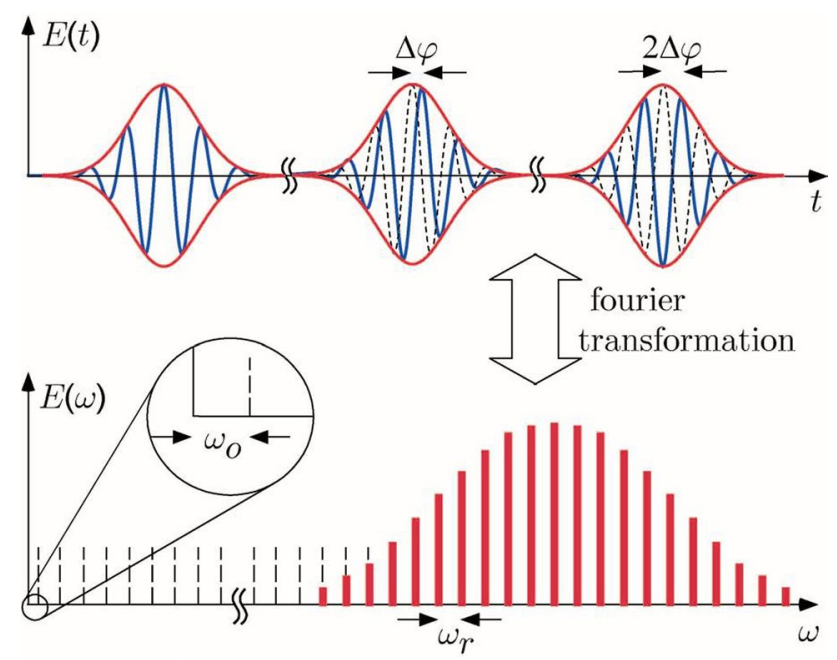

Fig. 1 Electric field versus time (upper panel) and its Fourier transform (lower panel) in a mode-locked laser, as observable at a fixed position on the resonator axis. Courtesy of Thomas Udem 
Fig. 2 Laser system producing the first intense, waveform-controlled light pulses. For more details, see text and Ref. [18]

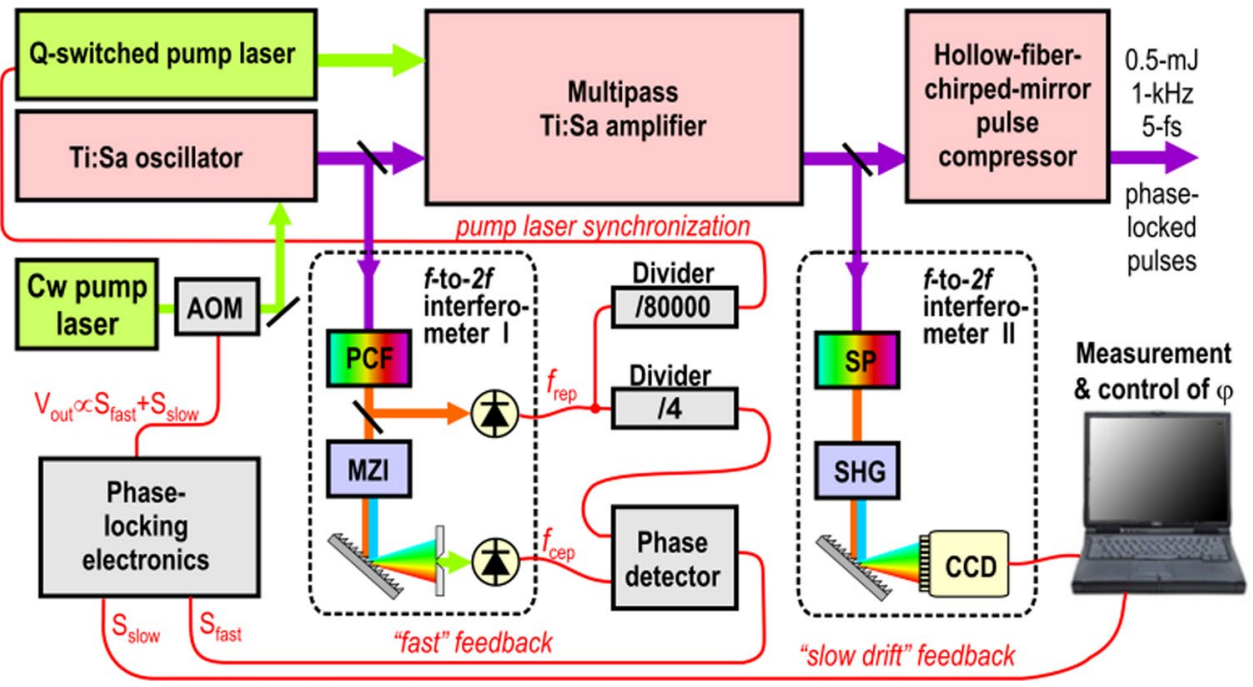

on the optical axis observes a periodically reappearing pulse with identical $\mathbf{E}_{\text {peak }}, a_{L}(t)$, and $\omega_{L}$ but with a carrierenvelope (CE) phase, $\varphi$, changed by $\Delta \varphi$ as given by (2).

This quasi-periodic train has a frequency spectrum that consists of a series of equidistant lines, with their frequency given by

$\omega_{n}=\omega_{0}+n \omega_{r}$,

where $\omega_{r}=2 \pi / T_{R}$ is the round-trip frequency and $n$ is a large $\left(\sim 10^{6}\right)$ integer. The offset frequency $\omega_{0}$ is connected to $\Delta \varphi$ by the simple relationship [4]

$\omega_{0}=\frac{\Delta \varphi}{T_{R}}$.

We demonstrated that the round-trip CE phase shift $\Delta \varphi$ can be adjusted by a component of variable dispersion, such as a pair of glass wedges [16]. However, this manual control of $\Delta \varphi$ is insufficient for laser pulse generation with precisely reproduced field evolution. This is because small fluctuations in $\mathbf{E}_{\text {peak }}, a_{L}(t)$, and $\omega_{L}$ translate into perturbations to $\varphi$. These small perturbations typically remain nearly constant over many round trips allowing their accumulation to substantial random shifts of $\varphi$ over multiple round trips [16]. Therefore, control over the electric field evolution of the pulses emitted by a mode-locked laser requires active stabilization of the $\mathrm{CE}$ phase.

Equation (4) reveals that control over the CE phase calls for controlling the offset frequency $\omega_{0}$. To this end, $\omega_{0}$ has to be converted into an electronic signal. This was accomplished by the self-referencing technique invented by Theodor Hänsch. The conceptually simplest implementation of the method was first described and published in Ref. [14]. The low-frequency end of an octave-spanning mode-locked spectrum is frequency doubled and brought into interference with the high-frequency end of the same spectrum. The resultant beating occurs at a frequency of $2\left(n \omega_{r}+\omega_{0}\right)-\left(2 n \omega_{r}+\omega_{0}\right)=\omega_{0}$ and yields the required intensity modulation at $\omega_{0}$, which can be easily measured by a photodiode 10,17 . The approach has been dubbed the f-to-2f technique. Phase locking $\omega_{0}$ obtained this way to a sub-harmonic of the round-trip frequency, $\omega_{r} / N$, yieldsaccording to (4) $-\Delta \varphi=2 \pi / N$ and hence a pulse train in which every $N$ th pulse has identical CE phase and-assuming constancy of all other pulse parameters-thereby identical electric field waveforms. This approach resulted in the first waveform-controlled few-cycle (sub-10-fs) pulses from a Ti:sapphire oscillator [17].

\section{Strong, controlled, few-cycle waveforms}

Few-cycle pulses from the Ti:sapphire oscillator carry only nanojoule energies, far too low to produce field strengths needed for strong-field-induced electron phenomena. The controlled waveforms from the oscillator therefore need to be amplified, and possible random shifts and drift of the CE phase introduced in the amplifier system need to be compensated. Figure 2 outlines the system developed to this end by Andrius Baltuska, Matthias Uiberacker, Michael Hentschel and co in Vienna in cooperation with Christoph Gohle, Ronald Holzwarth, and Thomas Udem from Garching, which delivered intense laser pulses with controlled field oscillations for the first time [18]. The octave-spanning spectrum of the pulses produced by the oscillator as well as the pulse compressor following the amplification allowed straightforward implementation of the f-to- $2 \mathrm{f}$ technique both for controlling the evolution of $\varphi$ in the oscillator output and for stabilizing $\varphi$ of the amplified pulses compressed to a duration of $5 \mathrm{fs}$ (full width at intensity half maximum). 


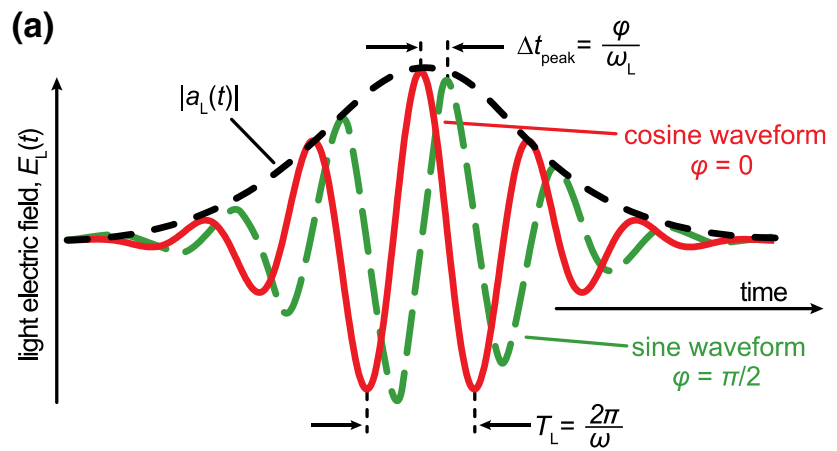

(b)

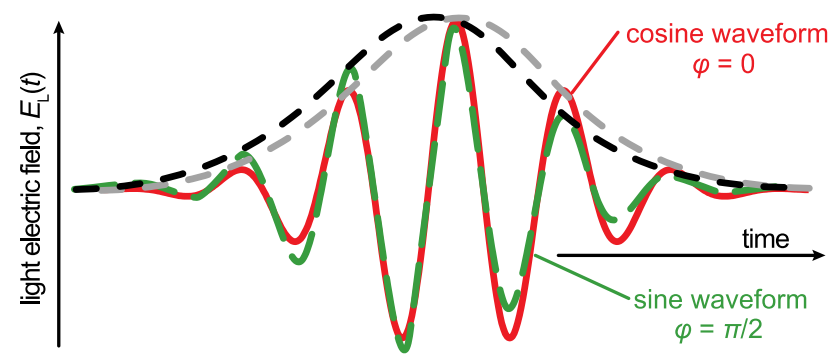

Fig. 3 Few-cycle waveforms with different CE phase, shown with the peak of their amplitude envelope (panel $a$ ) and the zero transitions of their field oscillations (panel $b$ ) coinciding

The system permits generation of this few-femtosecond duration, few-cycle pulses with arbitrary setting of the CE phase. Figure 3a depicts two representative waveforms with most distinct differences, the "cosine" waveform with a single most intense half cycle at the center of the pulse and the "sine" waveform with two equally strong half cycles of opposite direction separated by a zero crossing of the field at the pulse center. In this representation (coinciding pulse envelopes), the two waveforms appear quite different but comparing them with their zero crossings coinciding (Fig. 3b) reveals only minor deviations between their field evolutions. However, even these miniscule differences can have dramatic ramifications when it comes to nonlinear field-electron interactions.

\section{Attosecond control}

A highly nonlinear response to a few-cycle excitation offers the potential for confining the effective light-electron interaction to a single wave cycle. High-order harmonic emission constitutes a textbook example for an electronic process responding to the driving electric field in an extremely nonlinear fashion [19]. As illustrated in Fig. 4, the peak intensity of a few-cycle laser pulse can be adjusted such that the electric field is strong enough to liberate the most weakly bound electron of an atom only at the oscillation peak(s) of the central, most intense wave cycle with

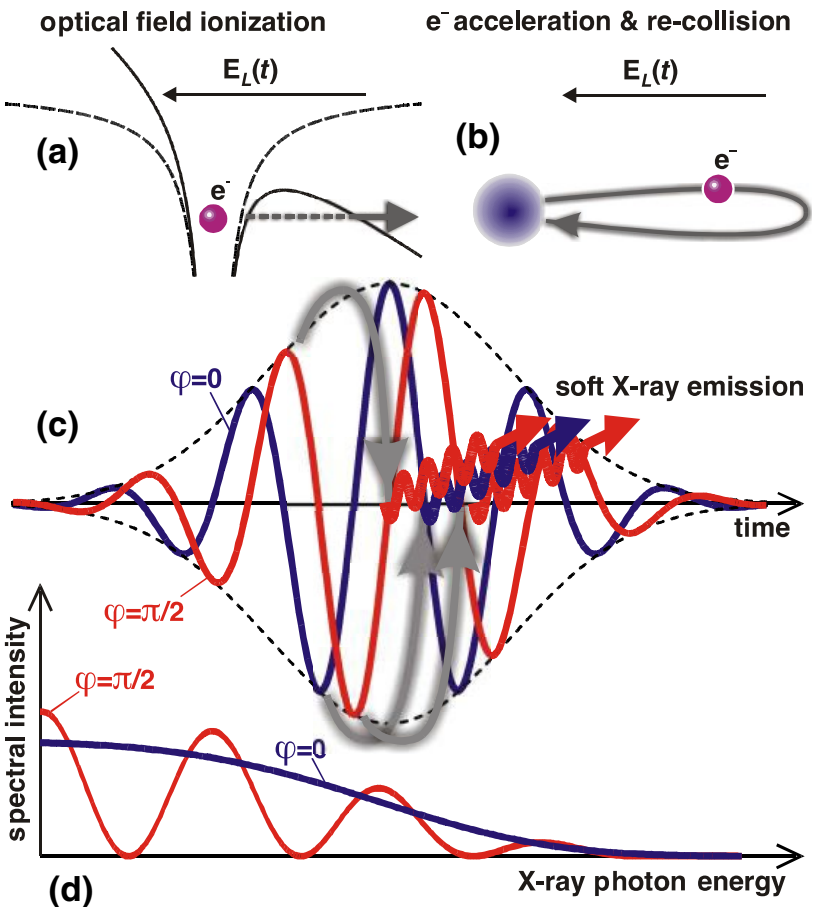

Fig. 4 XUV emission from optical field ionization (also known as high-order harmonic generation when induced by multi-cycle optical fields). a Liberation of most weakly bound electron by tunneling ionization. b Acceleration of the freed wavepacket by the linearly polarized laser field, recollision of the wavepacket with its parent ion. $\mathbf{c}$ Emission of XUV radiation upon recollision, for cosine- (blue) and sine (red)-shaped few-cycle excitation

substantial probability. The liberated electron is initially pulled away from its original atomic location and-after a brief excursion in the strong laser field-pushed back to its parent ion. Here, interference of the returning wavepacket with the bound-state portion of the wavefunction of the same electron gives rise to high-frequency charge oscillations. The resultant XUV burst emitted by this tiny atomic dipole antenna is expected to last a fraction of the field half cycle, i.e., a fraction of a femtosecond.

Once this process is confined to a single wave cycle, the apparently tiny (see Fig. 3b) difference between a cosine and sine excitation waveform has striking implications. As a matter of fact, differing temporal symmetry results in different system response: the cosine waveform is expected to give rise to the emission of a single sub-femtosecond burst of XUV light, whereas the sine-shaped driver should yield-for symmetry reasons-twin pulses. The spectral distribution of the emitted radiation provided a first clear indication of this picture being correct. The high-energy end (cutoff region) of the XUV spectra in Fig. 5 summarizing experimental results for different driving waveforms reveals a quasiperiodic modulation and an unmodulated continuum for sine and cosine excitation, respectively. This is exactly the behavior expected 


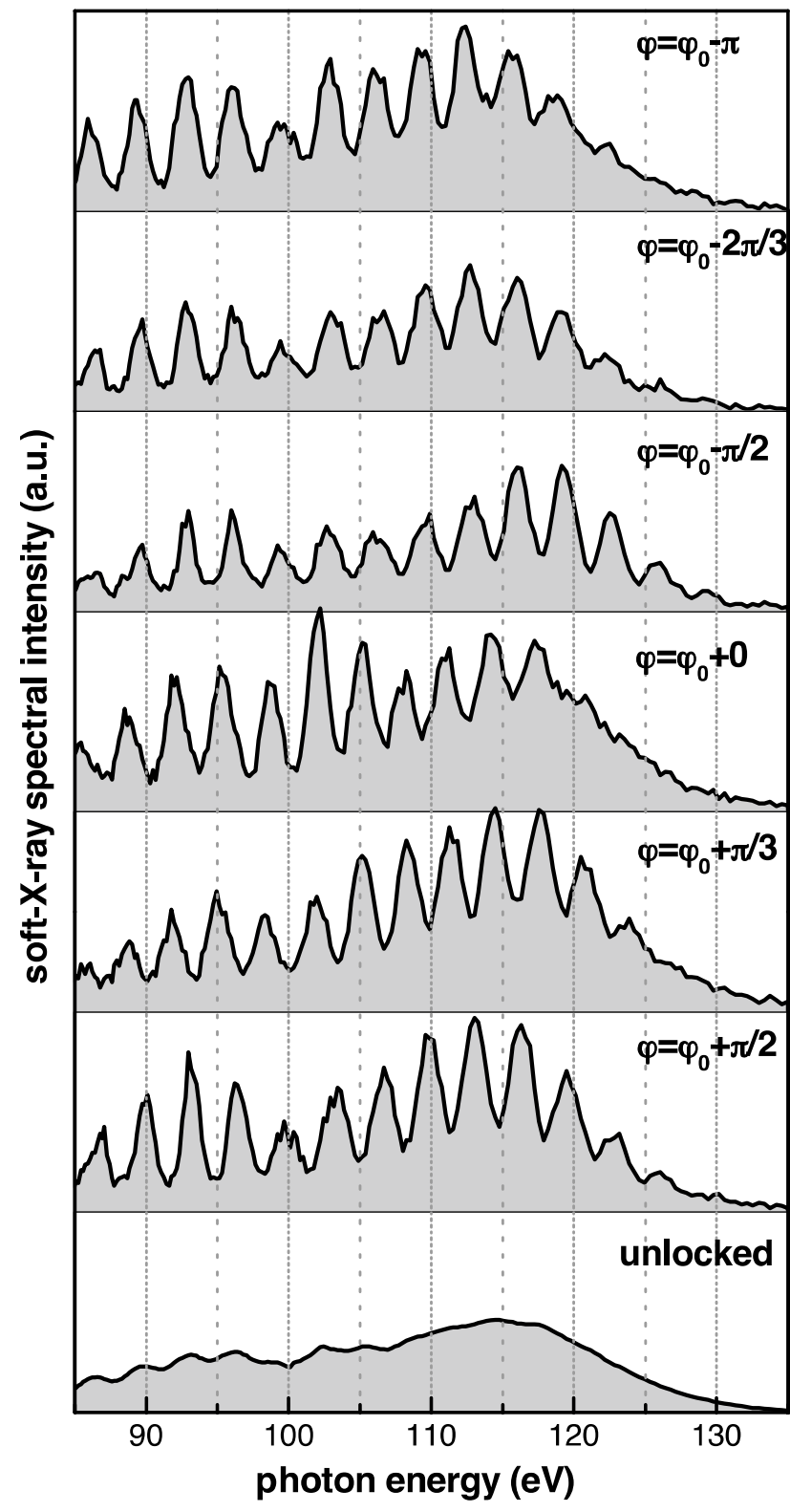

Fig. 5 Spectra of XUV emission emerging from neon atoms ionized by linearly polarized few-cycle laser pulses of different $\mathrm{CE}$ phase. For more details, see text and Ref. [18]

for the emission of twin and single bursts, respectively. To obtain more direct evidence about the temporal structure of the emitted high-frequency radiation, an attosecond-resolution metrology is in demand. Once again, light waveform control is the key to meet this demand.

\section{Attosecond metrology}

The sub-laser-cycle XUV emission emerging from the ionization process described above is locked to the laser

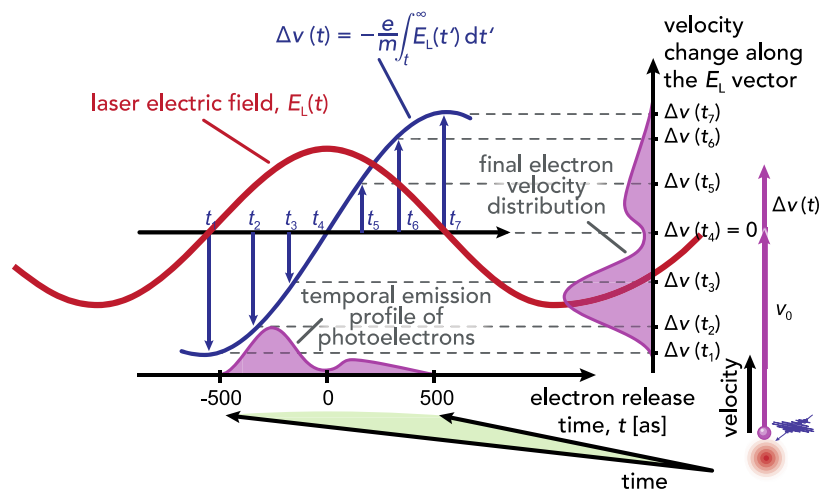

Fig. 6 Basic concept of an attosecond streak camera, see text for explanation

field oscillation driving the process. This attosecond synchronism offers the potential for using the generating laser field also for the measurement (temporal characterization) of the XUV light. Because the laser field can't directly interact with another light field, the temporal profile of the XUV light needs to be reproduced with high fidelity in some electronic process. To this end, a second set of atoms are exposed simultaneously to the XUV and laser light. The XUV photons liberate the most weakly bound electron of some of the atoms in a process that has been thought instantaneous. Meanwhile, precision attosecond metrology revealed a delay of this photoemission process. This delay is of the order of $10^{-17} \mathrm{~s}$ and does not affect the temporal profile of the emission significantly. Hence, the (hypothetical) temporal emission profile of the emitted photoelectrons shown in Fig. 6 mimics that of the incident XUV light.

This temporal profile can be captured by using the strong, controlled electric field of the laser field which produced the XUV pulse a few nanoseconds earlier (in another set of atoms) as a "chronoscope." The laser field changes the initial velocity of the freed electron by an amount proportional to the vector potential of the field at the instant of photoelectron ejection. For an emission process confined to a fraction of the half cycle of the laser field, the change in electron velocity varies near linearly with the emission time, projecting the temporal profile of the emission to a corresponding final velocity distribution of photoelectrons as illustrated in Fig. 6. Even if this linearity condition is not fulfilled, the temporal emission profile can be unambiguously and accurately retrieved from recording several XUV-induced and "laser-streaked" photoelectron spectra at different timing of the XUV burst with respect to the "streaking" laser field.

The method has been dubbed laser-field-driven (or briefly attosecond) streaking and constitutes, ever since its first demonstrations (in 2001 and 2004 without and with 


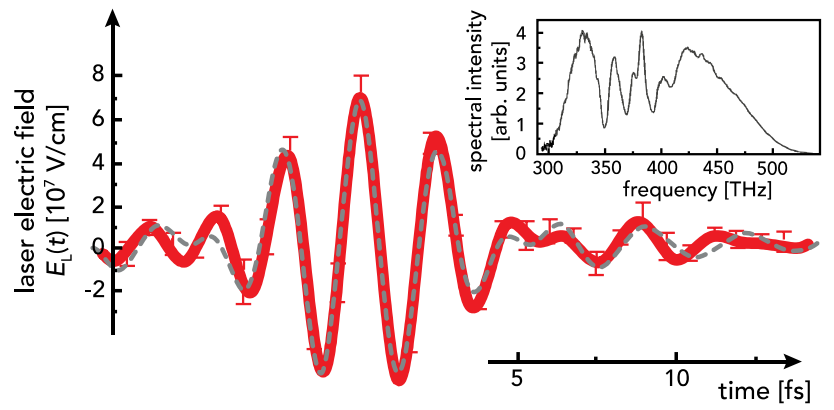

Fig. 7 First light pulse with its waveform measured. For details, see Ref. [21]

waveform-controlled laser light, respectively) [20, 21], the gold standard in attosecond metrology. Attosecond streaking permits much more than just measuring the duration of attosecond pulses, which can meanwhile be produced with durations shorter than 100 as. With the timing of the attosecond pulses being scanned across the entire few-cycle laser field in the light-field-driven streak camera, the evolution of the laser field can also be retrieved. Figure 7 shows the first measured light waveform, which was obtained this way [22].

With the attosecond pulse and its few-cycle driver laser field fully characterized, attosecond streaking and pumpprobe approaches constitute a powerful set of approaches for interrogating ultrafast electron phenomena occurring in excited atomic systems. With the attosecond pulse as a trigger, laser-field-driven streaking can be used as an attosecond chronoscope for timing photoemission from different atomic states or measuring electron propagation delay in solids [23]. With the fully characterized oscillating laser field as probe, one can trace the nonlinear polarization response of matter to optical fields [24]. The attosecond pulse, on the other hand, can be applied to probe strong-field-induced electron phenomena via attosecond transient absorption or photoelectron spectroscopy [23]. With these tools and techniques, attosecond metrology and spectroscopy are now well established and being used in a rapidly growing number of laboratories for studying electron dynamics.

\section{Future prospects: exploring electronic motions in complex systems}

The attosecond-varying force of visible-infrared waveforms along with attosecond-duration pulses of short-wavelength light synchronized to this force has enabled real-time observation (and-to some extent-control) of atomicscale electron dynamics for the first time. The physical observables captured with attosecond temporal resolution, such as photoelectron momentum, transient absorption, induced polarization, have provided-and continue to provide-unprecedented insight into the dynamic response of the electrons to external excitation.

However, there is another observable of crucial importance: the expectation value of the electrons' spatial coordinates. Dynamic changes in the electrons' atomic-scale distribution and the atomic-scale current densities associated with these changes play a pivotal role in chemical reactions underlying vital biological processes of living organisms as well as in the quest for ever-smaller and ever-faster electronic signal processing circuitry.

Whereas the electrons' spatially averaged excursion from their equilibrium (ground state) position has been accessed by attosecond polarization spectroscopy [23], atomic-scale rearrangements of electron charge distributions occurring on the electronic timescale remained inaccessible in complex systems, such as biological molecules and solid-state nanostructures, to this day. Making them perceivable requires attosecond-duration multi-keV photon (X-ray) or electron pulses, permitting diffraction imaging with picometer spatial and attosecond temporal resolution.

Again, controlled light forces may offer the solution. The synthesis of multi-octave, multi-terawatt visible-infrared optical transients holds promise for the generation of isolated attosecond pulses at several $\mathrm{keV}$ photon energies by ionizing an ensemble of helium atoms [25], whereas moderate-power, precisely controlled infrared transients may pave the way toward electron pulses with sub-femtosecond duration [26].

In conclusion, optical field synthesis, first enabled by the frequency-comb stabilization technique of Theodor Hänsch, has been playing a central role in establishing the basic techniques and tools for precision attosecond control and metrology and is likely to continue playing a pivotal role in its future evolution. There is plenty of room for further synergies between time- and frequency-domain metrologies, motivating the respective communities to continue their collaboration initiated by the pioneering research of Theodor Hänsch some 20 years ago.

Acknowledgement Open access funding provided by Max Planck Society. I gratefully thank Mandy Singh for her support in preparing this manuscript for publication.

Open Access This article is distributed under the terms of the Creative Commons Attribution 4.0 International License (http://creativecommons.org/licenses/by/4.0/), which permits unrestricted use, distribution, and reproduction in any medium, provided you give appropriate credit to the original author(s) and the source, provide a link to the Creative Commons license, and indicate if changes were made. 


\section{References}

1. T.W. Hänsch, Nobel lecture: passion for precision. Rev. Mod. Phys. 78, 1297 (2006)

2. A.H. Zewail, Femtochemistry: atomic-scale dynamics of the chemical bond. J. Phys. Chem. A 104, 5660 (2000). (Nobel Lecture)

3. F. Krausz, The birth of attosecond physics and its coming of age. Phys. Scr. 91, 063011 (2016)

4. T. Udem, Phasenkohärente optische Frequenzmessungen am Wasserstoffatom. Thesis, Ludwig-Maximilians Univ (1997)

5. J. Reichert, R. Holzwarth, T. Udem, T.W. Hänsch, Measuring the frequency of light with modelocked lasers. Opt. Commun. 172, 59 (1999)

6. Th Udem, J. Reichert, R. Holzwarth, T.W. Hänsch, Accurate measurement of large optical frequency differences with a modelocked laser. Opt. Lett. 24, 881 (1999)

7. Th Udem, J. Reichert, R. Holzwarth, T.W. Hänsch, Absolute optical frequency measurement of the cesium $D 1$ line with a mode-locked laser. Phys. Rev. Lett. 82, 3568 (1999)

8. H.R. Telle, G. Steinmeyer, A.E. Dunlop, D.H. Sutter, U. Keller, Carrier-envelope offset phase control: a novel concept for absolute optical frequency measurement and ultrashort pulse generation. Appl. Phys. B 69, 327 (1999)

9. D.J. Jones et al., Carrier-envelope phase control of femtosecond mode-locked lasers and direct optical frequency synthesis. Science 288, 635 (2000)

10. S.A. Diddams et al., Direct link between microwave and optical frequencies with a $300 \mathrm{THz}$ femtosecond laser comb. Phys. Rev. Lett. 84, 5102 (2000)

11. J. Reichert et al., Phase coherent vacuum-ultraviolet to radio frequency comparison with a modelocked laser. Phys. Rev. Lett. 84, $3232(2000)$
12. M. Niering et al., Measurement of the hydrogen $1 S-2 S$ transition frequency by phase coherent comparison with a microwave cesium fountain clock. Phys. Rev. Lett. 84, 5496 (2000)

13. R. Holzwarth et al., Optical frequency synthesizer for precision spectroscopy. Phys. Rev. Lett. 85, 2264 (2000)

14. J. Ye et al., Accuracy comparison of absolute optical frequency measurement between harmonic generation synthesis and a frequency division femtosecond-comb. Phys. Rev. Lett. 85, 3797 (2000)

15. T. Udem, R. Holzwarth, T.W. Hänsch, Optical frequency metrology. Nature 416, 233 (2002)

16. L. Xu et al., Route to phase control of ultrashort light pulses. Opt. Lett. 21, 2008 (1996)

17. A. Apolonski et al., Controlling the phase evolution of few-cycle light pulses. Phys. Rev. Lett. 85, 740 (2000)

18. A. Baltuska et al., Attosecond control of electronic processes by intense light fields. Nature 421, 611 (2003)

19. X.F. Li, A. L'Huillier, M. Ferray, L.A. Lompre, G. Mainfray, Multiple-harmonic generation in rare gases at high laser intensity. Phys. Rev. A 39, 5751 (1989)

20. M. Hentschel et al., Attosecond metrology. Nature 414, 509 (2001)

21. R. Kienberger et al., Atomic transient recorder. Nature 427, 817 (2004)

22. E. Goulielmakis et al., Direct measurement of light waves. Science 305, 1267 (2004)

23. F. Krausz, M. Ivanov, Rev. Mod. Phys. 81, 163 (2009)

24. A. Sommer et al., Attosecond nonlinear polarization and lightmatter energy transfer in solids. Nature 534, 86 (2016)

25. H. Fattahi et al., Third-generation femtosecond technology. Optica 1, 45 (2014)

26. C. Kealhofer et al., All-optical control and metrology of electron pulses. Science 352, 429 (2016) 